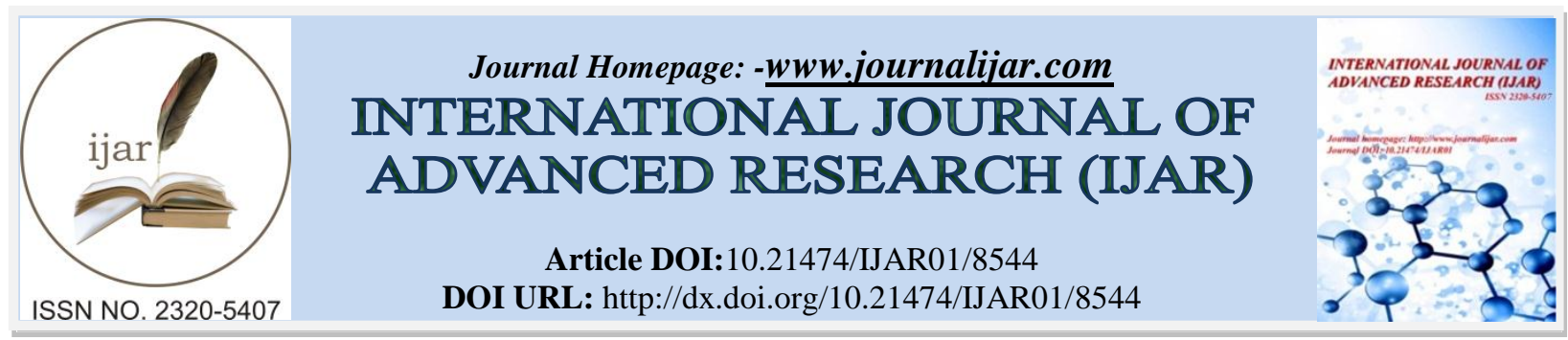

RESEARCH ARTICLE

\title{
CARDIAC AUTONOMIC NEUROPATHY EXPERIENCE OF DEPARTMENT OF CARDIOLOGY A, IBN SINA UNIVERSITY HOSPITAL CENTER, RABAT, MOROCCO.
}

Lalla Fatima Ezzahra El Hassani, Salwa Cheraou, Halima Benjelloun Rokya Fellat and Mohamed Cherti. Department of Cardiology A, Ibn Sina University Hospital Center, Rabat, Morocco.

\section{Manuscript Info}

\section{Manuscript History}

Received: 11 December 2018

Final Accepted: 13 January 2019

Published: February 2019

Key words:-

Blood pressure, Cardiac autonomic neuropathy, Diabetes mellitus, Heart rate.

\begin{abstract}
Introduction: Cardiac autonomic neuropathy (CAN) is a common complication of diabetes mellitus, which is associated with a higher risk of morbidity and mortality. It can be detected by analyzing spontaneous (Holter) or provoked (Ewing's test battery) changes in heart rate and arterial blood pressure. Baroreflex gain is a specific index of great interest.

Methods: Through the experience of the team exploring the autonomic nervous system within the Ibn Sina University Hospital Center , this study approached CAN in 60 diabetic patients collected during the period from 2015 to 2017, thanks to the use of different tests of Edwing .

Results: The duration of diabetes significantly influences the occurrence of a CAN ( $\mathrm{p}=0.002)$, also the overweight $(\mathrm{p}=0.002)$, arterial hypertension $(0.005)$.Clinical symptomes were mainly orthostatic hypotension, dyspnea and palpitation.The autonomic profile among the patients was dominated by exaggerated responses of the alpha sympatic a system .

Conclusions: Our study confirms the high frequency of CAN in diabetics. The main risk factors associated with it are hypertension and overweight. The presence of an orthostatic hypotension testifies to the severity of the CAN. The coexistence of hypertension in these patients worsens its prognosis and makes it easy to manage.The diagnosis of CAN is important for the follow-up of these patients while waiting for more specific treatments.
\end{abstract}

Copy Right, IJAR, 2019,. All rights reserved.

\section{Introduction:-}

Cardiac autonomic neuropathy (CAN) is very common in the diabetic population. Often revealed at the infra-clinical stage, it can however be at the origin of serious complications. Its screening is simple and is done through the exploration of the autonomic nervous system through the collection of simple parameters such as heart rate and blood pressure and their analysis to assess the sympathetic and parasympathetic function.

\section{Objective:-}

The objective of our study is to determine the autonomic profile of diabetics and describe their symptoms and associated risk factors.

Corresponding Author:-Lalla Fatima Ezzahra El Hassani.

Address:-Department of Cardiology A, Ibn Sina University Hospital Center, Rabat, Morocco. 


\section{Material and method:-}

This is a retrospective study of 60 type 1 and type 2 diabetic patients, explored within the autonomic nervous system unit of the cardiology department A at the IBN SINA Ibn Sina University Hospital Center of RABAT between the year 2015 and 2017.

The autonomic nervous system (ANS) tests are performed on an empty stomach after stopping all treatment for at least 48 hours. Patients are first placed in a quiet, supine position with monitoring of blood pressure and heart rate. These parameters are measured at rest every 5 minutes for 30 minutes.

Subsequently we proceeded to the various tests as described by EWING:

1. Forced breathing or Deep breathing test.

2. Hand grip

3. Hyperventilation

4. Mental stress

5. Orthostatism test.

\section{Results:-}

\section{Epidemiology:}

The age of our patients is between 16 and 79 years, with an average age of 55 years. $58 \%$ of the patients were female.

\begin{tabular}{|c|c|c|}
\hline AGE RANGE & NUMBER OF PATIENTS & $\%$ \\
\hline$<20$ & 1 & 1,67 \\
\hline $20-40$ & 6 & 10,00 \\
\hline $40-60$ & 34 & 56,67 \\
\hline$>60$ & 19 & 31,67 \\
\hline
\end{tabular}

Table $\mathbf{n}^{\circ}$ 1:-Repartition of patients by age group

\section{Diabetes:}

In our study only four patients had type 1 diabetes, three of which were male.

The seniority of diabetes is not known in all our patients. However, there was a minimum duration of 2 years and a half and a maximum of 35 years. Diabetes was poorly balanced in the majority of patients. Micro or macroangiopathic complications were associated in $30 \%$ of them.

\section{Risk Factors Associated:}

1-Obesity: \%

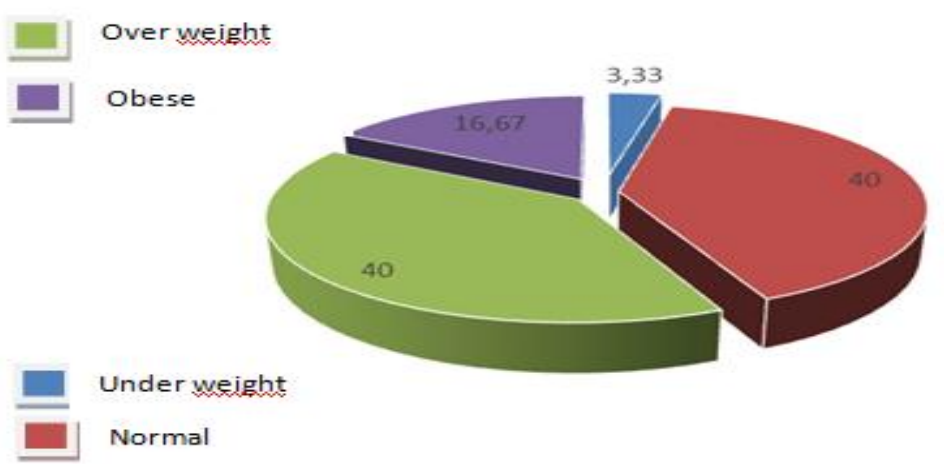

Figure $\mathbf{n}^{\circ}$ 1:-Distribution of body mass index among patients 
2-Arterial hypertension:

$75 \%$ of patients in our series were known hypertensive and under treatment.

3-Dyslipedemia:

Total cholesterol: $65 \%$ of patients had higher cholesterolemia

Triglyceride: $59 \%$ of patients had higher triglyceridemia

High density lipoprotein (HDL): $43 \%$ of hypo HDL cases

Low density lipoprotein (LDL) : $49 \%$ of them in hyper LDL

\section{Clinical Manifestations:}

Clinical examination was normal in all patients.

Clinical symptoms presented by patients:

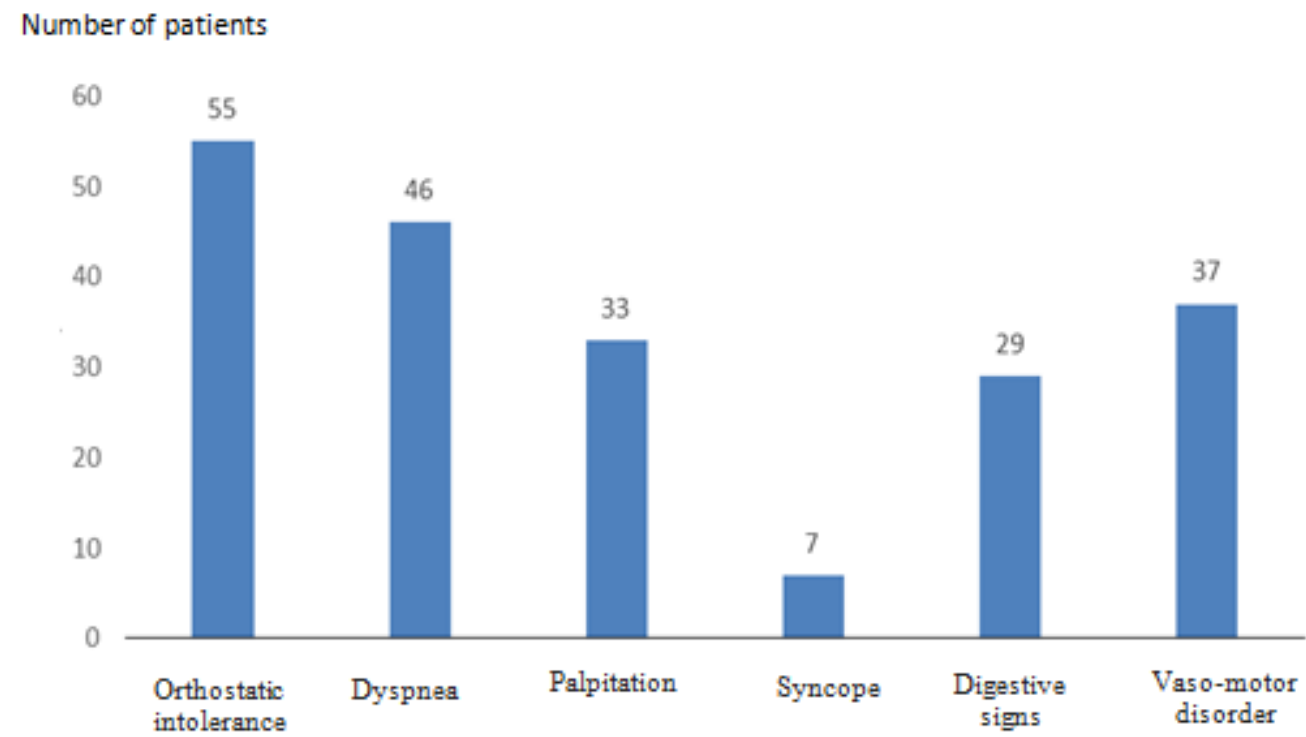

Autonomous Profile:

Figure $\mathbf{n}^{\circ}$ 2:-Clinical symptoms presented by patients

One-third of patients in our series had vagal impairment

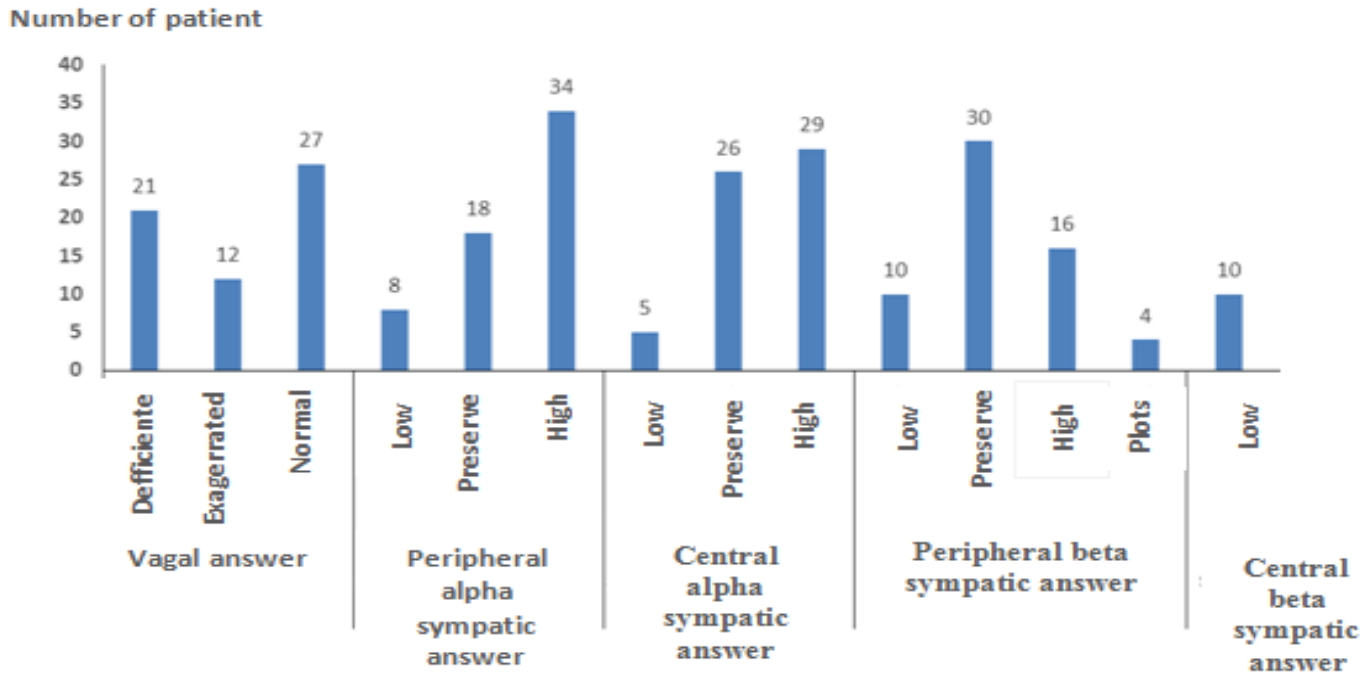

Figure n ${ }^{\circ}$ 3:-Autonomic profil of our diabetic patients 


\section{Discussion:-}

Cardiac autonomic neuropathy $(\mathrm{CAN})$ is one of the most common complications of diabetes mellitus. However, it remains the least practically studied and appears as a "poor relative" in comparison with the interest in other complications of micro and macro-angiopathy (nephropathy, retinopathy, coronary artery disease). Indeed, despite a high prevalence and an obvious relationship with the occurrence of cardiovascular events, its systematic search, precise quantification and, even more, its treatment remain largely insufficient. Some explanations for this lesser impact (on doctors and, therefore, on patients) of this complication lie, at least in part, in a certain lack of harmonization in its diagnosis and in the uncertainties regarding supported.

CAN is defined as an abnormality of the autonomic nervous system, sympathetic and parasympathetic, and can be recognized on the basis of clinical elements or "subclinical" (positive screening tests).

CAN is the result of the dysfunction of the small amyelic fibers of the ANS innervating the heart and blood vessels [1]. This leads to a decrease of the parasympathetic tonus during the night in favor of the sympathetic activity which could consequently expose to cardiovascular events. Metabolic (polyol pathway) and microvascular factors have been implicated in the pathophysiology of CAN.

Schematically we can divide the CAN into peripheral lesions, affecting mainly the lower limbs (distal sensitivomotor polyneuritis), and autonomic attacks, then affecting the different systems (cardiovascular, digestive, urogenital and even bronchopulmonary) [2] [3].

The prevalence of CAN varies from $2.4 \%$ in a population newly diagnosed with diabetes to more than $36 \%$ in some large cohorts of diabetic patients. Tests based on cardiac frequency variation are positive in 20 to $60 \%$ of the diabetic population and imply that the presence of a CAN largely precedes the occurrence of orthostatic hypotension and its attendant symptoms [4]. The occurrence of CAN is sometimes seen in the first years after the onset of diabetes, and it is now established that CAN should not be perceived as a rare, late, and inconsequential complication in the evolution of the diabetic subject.

It is also one of the main dysautonomia especially in type 2 diabetics [1], the same finding was found in our study population; 57 patients had a type 2 diabetes. It reaches $6 \%$ of newly diagnosed diabetics and about $15 \%$ of patients whose diabetes has been known for less than one year [5]. In these patients, parasympathetic deficiency precedes sympathetic fiber involvement [6]. The presence of CAN is associated with higher mortality [7], hence the value of early diagnosis based on autonomic nervous system (ANS) exploration tests. In fact, this excessive mortality could be explained by a higher incidence of silent myocardial infarction, increased mortality after myocardial infarction, prolongation of the QT interval responsible for sudden

death and, finally, a higher risk of death,have nephropathy, presumably by renal hemodynamic disorder [8].

The duration of diabetes and the poor control of blood glucose levels are the main risk factors for CAN [9], as shown by the results found in our patients who had a minimum diabetes duration of 2 years and a half maximum of 35 years. poor balance of diabetic disease in $90 \%$ of patients. Indeed ; the duration of diabetes significantly influences the occurrence of a CAN: the stigmas of CAN are particularly important after 20 years of diabetes evolution, in addition to the poor glycemic balance of a few years ( 3 to 4 years) is sufficient to alter the baro-reflex gain.

Risk factors should be noted such as:

1. Overweight, $40 \%$ of patients were overweight $(\mathrm{p}=0.002)$

2. the advanced age, $56.67 \%$ of patients aged between 40 and 60 years, $31.67 \%$ were over 60 years old.

3. Arterial hypertension: $75 \%$ of patients in our series were known hypertensive and under treatment. Pulsed blood pressure, an independent cardiovascular risk factor, increases with the duration of diabetes, regardless of age $(\mathrm{p}=0.005)$

4. Dyslipidemia: is also a risk factor significantly associated with the onset of CAN .

5. The metabolic syndrome,

6. Smoking and alcoholism.

In our population, $\mathrm{CAN}$ is well explained by old diabetes, unbalanced and complicated $(\mathrm{p}=0.02)$ 
In practice, CAN screening is mandatory for the largest number of diabetics using at least two tests assessing variations in heart rate (Respiratory and Orthostatic). Once it is detected, additional management is required (search for orthostatic hypotension, silent myocardial ischemia, search for QT segment lengthening or paroxysmal ventricular rhythm disorder at ECG holter if QT lying on the basic ECG). In this situation, early detection of other complications of diabetes is required [10].

Depending on the degree of severity, the American Diabetes Association [11] defines CAN in 3 phases;

1. an early phase when there is an abnormality of heart rate at the Deep breathing,

2. an intermediate phase, if there is an anomaly of heart rate during the valsalva maneuver

3. and a severe phase when there is orthostatic hypotension.

The diagnosis of CAN, like that of peripheral neuropathy, can be suggested based on several symptoms and signs [12]. As our study also shows tiredness, weakness, dizziness, blurred vision and sometimes syncope after a transition from supine to standing are the signs of orthostatic hypotension. This is responsible for the vast majority of complaints raised by patients with CAN. Orthostatic hypotension, verified during a standard clinical examination, is however not pathognomonic of CAN and, moreover, its absence does not exclude the latter.

In CAN, orthostatic hyptension represents the major cardiovascular event [13]. In addition, orthostatic intolerance was the major symptom that prompted consultation in 55 patients in the study. Orthostatic hypotension was found in $28 \%$ of cases. His presence usually indicates severe cardiac autonomic neuropathy.

Orthostatic hypotension is defined as a decrease in systolic blood pressure $<20 \mathrm{mmHg}$ or PAD $<10 \mathrm{mmHg}$ after 03 minutes of orthostatism. It is classified in:

1. sympathicotonic orthostatic hypotension with an increase in heart rate of more than $15 \mathrm{bat} / \mathrm{min}$. It is a functional impairment of the vegetative baroreflex arc (anatomically intact ANS)

2. Neuropathic asympathicotonic orthostatic hypotension linked to an organic involvement of the ANS. It is accompanied by a decrease in the heart rate of less than $15 \mathrm{bat} / \mathrm{min}$

Orthostatic hypotension is due to the deficiency of the sympathetic answer, which is seen at a late stage after parasympathetic system involvement [14] and corresponds to a more severe grade [15]. The orthostatic test shows orthostatic hypotension.

Other cardiovascular complications are possible such as fixed tachycardia; 33 patients consulted for tachycardiarelated palpitations that were found on the electrocardiogram and peripheral vasomotor disorders found in 37 patients in the study. Attacks on other systems; not found in our patients, can be suspected in the presence of complaints suggestive of neurogenic bladder, erectile dysfunction, gastroparesis, often nocturnal motor diarrhea and repeated hypoglycaemia (favored by abolished adrenergic counter-regulation and erratic gastric emptying). [6] [1] [9]. In addition to clinical evidence, the diagnosis of CAN may be based on different screening tests. Simple tests make it possible to recognize the CAN at an early stage. These include the study of the loss of the variability of heart rate during standardized tests (forced breathing, Valsalva maneuver, orthostatism test)

Arterial hypertension in the presence of diabetes is very common [16] [17]. The prevalence of arterial hypertension was estimated at $53 \%$ in diabetic patients in a recent study [12], at $75 \%$ in our study population.

The combination of orthostatic hypotension and decubitus arterial hypertension in diabetics is also common [15]. To find out the prevalence of orthostatic hypotension in these diabetic hypertensive patients, one study showed that orthostatic hypotension was present in $20 \%$ of hypertensive diabetic patients and in only $4 \%$ of normotensive diabetic subjects [18]. This finding was also found in the study by el bekkali and al [17].

In practice, we can not really talk about treatment in the context of this complication of diabetes, but rather advice to implement to limit the clinical impact of CAN or new opportunities, certainly full of hope, but still in the research state. Indeed, since the pathophysiology of CAN is not clearly understood, it is not currently possible to propose an etiological treatment. Various practical measures have already been mentioned and remain important for reducing problems related to orthostatic hypotension [19]. They include mechanical approaches (wearing compression stockings, for example) or pharmacological (9-alpha-fluohydrocortisone to increase blood mass.) More experimental, several molecules have been studied or are still under investigation in the hopefully help patients with severe CAN, such as midodrine and other sympathomimetics such as ephedrine and $\alpha$-agonists may be helpful, 
agents such as clonidine or yohimbine may be useful in autonomous decompensation and Erythropoietin is also being studied as a possible treatment for orthostatic hypotension (thanks to the increase in blood volume) and finally, neurosurgical techniques attempting to improve the baro-reflex system are being investigated [20] [21] and favorable effects have sometimes been reported after electro-acupuncture sessions [22]. In our patients, it has been proposed:

1. Stop antihypertensive treatment. If systolique blood pressure in Dorsal Decubitus $>170 \mathrm{mmHg}$, short-acting antihypertensive therapy should be prescribed

2. Progressive lifting

3. Adequate water intake without salt intake

4. Peripheral venous restraint

5. veinotonic

6. Mididrine

Close monitoring of blood pressure is required for this type of patient.

In second intension, and if one is not effective on orthostatic hypotension, one had recourse to fludrocortisone

\section{Conclusion:-}

Our study confirms the high frequency of CAN in diabetics. The main risk factors associated with it are hypertension and overweight. Its screening is simple and must be performed on a large scale by practical, reliable, and non-invasive autonomic nervous system exploration tests to stratify the cardiovascular risk in these patients. The presence of an orthostatic hypotension testifies to the severity of the CAN. The coexistence of hypertension in these patients worsens its prognosis and makes it easy to manage.

The prevalence and severity of CAN in this population depend on the duration of the disease as well as the glycemic balance (a few years of poor control are sufficient to develop or worsen a CAN) for example. It is possible to establish different combinations of diagnostic tests that can identify this complication even at a very early stage. This diagnosis is important for the follow-up of these patients (the importance of a good metabolic balance and special attention to certain other risk factors), while waiting for more specific treatments.

\section{References:-}

1. Schumer MP,Joyner SA, and PfaiferMA.Cardiovascular autonomic neuropathy testing inpatients with diabetes.Diabetes Spectrum,1998.11:p.227-231

2. Scheen AJ.- La neuropathie diabétique : données épidémiologiques et prédictives. Diab Metab, 1998,24 (suppl 3), 45-49.

3. Dive D, Lievens I, Moonen G,Wang FC.- La neuropathie diabétique périphérique. Rev Med Liège, 2005, 60, 490-497.

4. Ziegler D, Dannehl K, Muhlen H, et al.- Prevalence of cardiovascular autonomic dysfunction assessed by spectral analysis, vector analysis, and standard tests of heart rate variation and blood pressure responses at various stages of diabetic neuropathy. Diabet Med, 1992, 9, 806814.

5. Valensi P, et al. Neuropathie autonome chez le diabétique. Recommandations del'ALFEDIAM. Diabètes Metab.1997.23(1) :p.89-99

6. Watkins PJ and Kitamura H, and Edmonds ME . Sympathetic nerve failure in diabetes. Diabétologia.1983.25 :p.73-7

7. Maser RE et al. The association between cardiovascular autonomic neuropathy and mortality in individuals with diabetes : a meta-analysis. Diabetes Care, 2003. 26 :p.1895-901

8. Maser RE, Mitchell BD, Vinik AI, et al. - The association between cardiovascular autonomic neuropathy and mortality in individuals with diabetes: a meta-analysis. Diabetes Care, 2003, 26, 1895-1901.

9. ToyryJP,etal.Occurrence, predictors, and clinical significance of autonomic neuropathyin NIDDM. Ten-year follow-up from the diagnostic, 1996.45 :p.308-15

10. Nevzat B et al,Increasing severity of cardiac autonomic neuropathy is associated withincreasing prevalence of nephropathy, retinopathy, and peripheral neuropathy in Turkish type 2 diabectics. Journal of diabetes and Its Complications, 2008.22 p.181-185

Boulton AJM et al. Diabetic neuropathies : a statement by the American Diabetes Association Diabetes Care,2005. 28 :p.956-62 
11. Low PA, Benrud-Larson LM, Sletten DM, et al.- Autonomic symptoms and diabetic neuropathy : a population-based study. Diabetes Care, 2004, 27, 2942-2947

12. Valensi P, La neuropathie autonome diabétique : quels sont les risques ?Diabetes andmetabolims, 1998.24 (suppl.3) :p.66-72

13. Benjelloun Ha, et al.Cardiovascularautonomic reflexes on the postural orthostatictachycardiasyndrome.Annales de Cardiologie et d'angéologie,2009. 58 :p.20-26

14. ValensiP,Gautier JE, AmarencoG, Sauvanet JP, leutengger M, Attali JR. :La neuropathie autonome chez le diabétique. Diabéte et métabolisme $1997 ; 23: 1-8$

15. Senard JM and Montastruc JL, Adrenoreceptor regulation in orthostatic hypotension during autonomic failure. Trends in Phramacological Sciences, 1993.14(10) :p.349-351

16. Mustapha El Bakkali, Halima Benjelloun, and HananRkain et al, A Cross-SectionalStudyEvaluatingOrthostatic Hypotension in Normotensive and Hypertensive Patients withDiabetesMellitus. Journal of cardiovasculardesease, 2013

17. MasuoK et al.Changes in frequency of orthostatic hypotension in elderly hypertensive patients under medications. American journal of hypertension,1996.9(3) :p.263-268

18. Scheen AJ.- Comment je traite...Un patient avec hypotension orthostatique. Rev Med Liège, 1997, 52, 134137.

19. Sato T, Kawada T, Sugimachi M, et al.- Bionic technology revitalizes native baroreflex function in rats with baroreflex failure. Circulation, 2002, 106, 730-4.

20. Yanagiya $\mathrm{Y}$, Sato $\mathrm{T}$, Kawada $\mathrm{T}$, et al.- Bionic epidural stimulation restores arterial pressure regulation during orthostasis. J Appl Physiol, 2004, 97, 984-990.

21. Zhou W, Fu LW, Tjen-A-Looi SC, et al-_ Afferent mechanisms underlying stimulation modality-related modulation of acupuncture-related cardiovascular responses. J Appl Physiol, 2005, 98, 872-880. 